\title{
Counting of Visible Foreign Particles in Liquid Injection Based on Trajectory Modelling
}

\author{
Sirui Wang, Qing Zhuo \\ Department of Automation, Tsinghua University, Beijing, \\ China \\ e-mail: zhuoqing@tsinghua.edu.cn
}

\author{
Ji’an Xia \\ Beijing xianquweifeng Technology Development Co., \\ Beijing, China \\ e-mail: xqwf_xja@126.com
}

\begin{abstract}
In order to meet the requirements of counting of visible foreign particles in liquid injection, an automatic visible foreign particles counting system based on trajectory modelling is developed. The mechanical part drives containers to spin and stop abruptly and afterwards the liquid continues to spin. A sequence of images is collected. Gaussian mixture model is used to detect moving targets. In the first step we track trajectories preliminarily by nearest neighbor approach and break unreasonable associations. In the second step, we model the particle movement by damped vibration and a SVM with modeling residuals as features was used to determine whether two short candidate tracks to belong to the same trajectory. Finally, we analyze the trajectories to determine the number of visible foreign particles.
\end{abstract}

Keywords-visible foreign particle; multiple targets tracking; nearest neighbor; support vector machine; trajectory modelling

\section{INTRODUCTION}

Visible foreign particle is insoluble matter that can be observed under prescribed conditions in liquid injection and the diameter of which is more than 50 microns by the standard of Chinese Pharmacopeia. In liquid injection used for human or livestock, visible foreign particles can cause serious harm. According to the medicine regulations, the number of visible foreign particles in liquid injection must be below a certain number, otherwise it should be discarded. Therefore, counting of visible foreign particles is important for medicine safety. Compared to counting method which is based on manual counting, an automatic method will achieve higher accuracy and efficiency. We developed a system that can count the number of visible foreign particles in liquid injection automatically based on trajectory modelling.

In our system, the mechanical part drives containers to spin and stop abruptly and afterwards the particles move with the liquid for a while. We obtain the accurate number of the particles by tracking. Tracking moving particles is essentially a multiple targets tracking problem. There are several major challenges involved in this application. (1) The appearance of the moving particles is small and weak. (2) The particles can be temporarily occluded by each other or the bottle walls. (3) Optical noise may cause false detection. (4) The particles are similar in appearance therefore tracking approaches using shape matching fail.

Several methods have been proposed to solving the problems. Veenman et al designed a framework based on the greedy optimal assignment which needs cost functions chosen artificially [1]. Statistical approaches such as JPDAF [2] and MHT [3] would take into account the uncertainty of measurement and model, but assumptions in this kind of approaches are usually strong and unable to be met in practical problems. Besides, statistical approaches are computationally demanding.

If a particle is occluded for quite a long time, it is almost impossible for previous approaches to track correctly. Our approach solves the problem by tracking particles hierarchically. First, nearest neighbor method is used to track particles preliminarily and acceleration constrains are used to break unreasonable tracks. Second, a SVM with $\mathrm{RBF}$ kernel is used to determine whether two candidate tracks should be linked to a complete trajectory. As shown in figure 1(a), in this application the particle moves in accordance with damped vibration on $\mathrm{x}$ axis which is used to model the $\mathrm{x}$ axis movement of the moving particles. We extract related features as input of the SVM. We can see from figure 1(b) that there are no strong characteristics on $y$ axis. We model the trajectory according to movement of the particles on $\mathrm{x}$ axis and the machine learning method applied can learn the characteristics of the specific problems by training and is more suitable for solving practical problems.
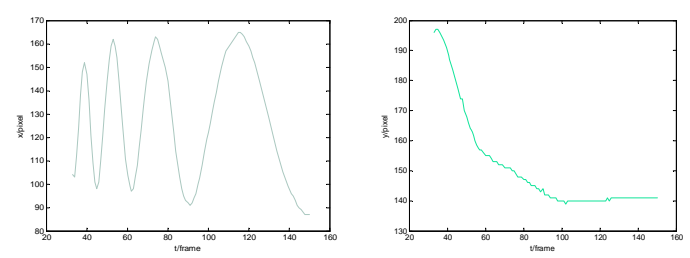

Figure 1. A typical particle movement trajectory: (a) trajectory on x axis.

(b) trajectory on y axis.

The outline of the paper is as follows: The next section introduces our system composition and how data are acquired. Then, in Section III, we present the whole process of multiple targets tracking and counting. We evaluate the approach with real data experiments in Section IV. Finally, we finish the paper with some conclusions in Section V. 


\section{SYSTEM COMPOSITION AND DATA ACQUISITION}

\section{A. System Composition}

The System hardware is mainly composed of three parts, namely, the mechanical part, the camera and the PC. The PC sends instruction to the mechanical part to make the container to spin for 2 seconds and then stop abruptly. After that the liquid continues to spin and the PC instructed the camera to collect images. This process lasts for 5 seconds and at the same time images are sent to the PC. The main process analyzes the images and giving the final number of the moving particles.

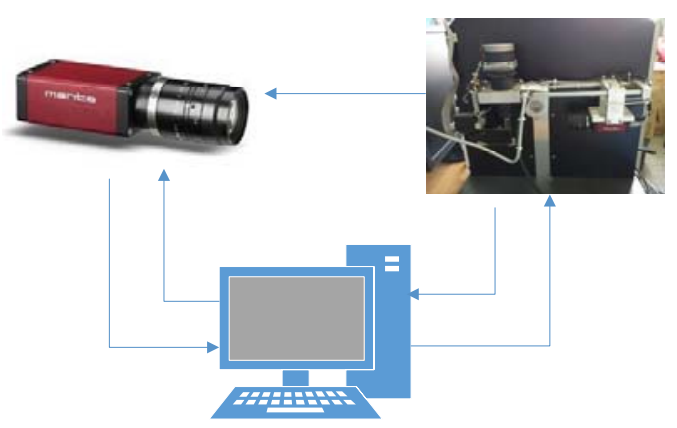

Figure 2. System composition.

\section{B. Data Acquisition}

In order to track trajectories, we need to find the positions of the moving particles in every frame. We acquire the data by moving particles detection. Gaussian mixture model is used to detect moving particles [4][5]. After processing each frame, a mask representing moving foreground is obtained. In the mask every connected domain is considered as a representation of a moving particle.
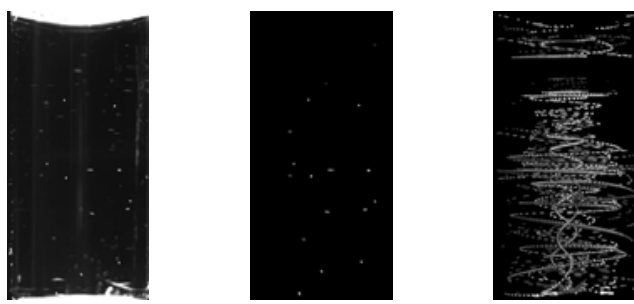

Figure 3. Moving particles detection: (a) Original frame. (b) Moving particles. (c) Moving particles superposition.

Now we have a set of data $X$ for a sequence of $n$ frames. The moving particle $p_{i}$ is on position $\mathbf{p}_{j}^{k}=\left(x_{j}^{k}, y_{j}^{k}\right)^{T}$ at time instance $t_{k}$ with $1 \leq k \leq n$ and $1 \leq j \leq m_{k} \cdot m_{k}$ is the number of detected moving particles in frame $\mathrm{k}$. For the kth frame, there is a correspondence set $P_{k}=\left\{\mathbf{p}_{j}^{k}\right\}$ representing detected moving particles. And the set
$X=\left\{P_{k}\right\}$ is a representation of all the detected moving particles in $\mathrm{n}$ frames. A track $T_{l}$ can be represented as $\left\langle\mathbf{p}_{j_{a}}^{a}, \cdots, \mathbf{p}_{j_{a+L-1}}^{a+L-1}\right\rangle$. Due to occlusion; some particle $p_{i}$ may have no correspondence position in some certain frames. Besides, due to false detection caused by noise, some position $\mathbf{p}_{j}^{k}$ may be correspondence to no moving particle.

\section{Multiple TARgets Tracking}

The proposed approach is hierarchical. In the first stage, nearest neighbor model is used and then acceleration constrains are used to break unreasonable track. In the second stage, we use a SVM to determine whether to link two segments of candidate tracks. We model the trajectory as damped vibration and use the residuals as features of the SVM.

\section{A. Nearest Neighbor Data Association}

Now we need to determine which two particle positions in two adjacent frames belonging to the same particle. Nearest neighbor approach is used.

A gate is formed around a particle position which belongs to a track in one frame, and every particle position falling within the gate in the next frame is considered as an association candidate. We choose the nearest position to the previous position as the particle position in the next frame and the track is one frame longer. If there is no positon falling in the gate, the track is interrupted. Positions in the next frame, which are not associated with existing tracks, generate new tracks. In our application the gate is a circle with a radius of 30 pixels.

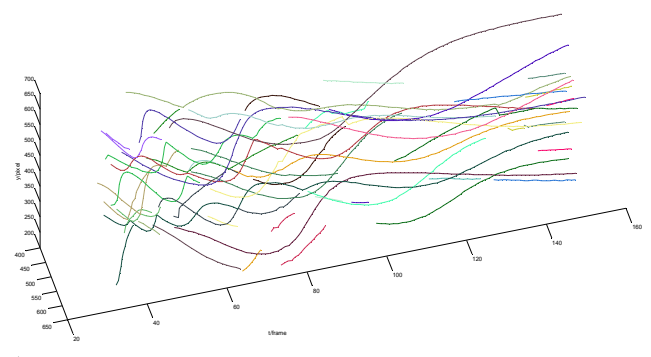

Figure 4. Nearest neighbor associations.

Nearest Neighbor approach is simple but degrades in clutter. If there are many moving particles, false associations are unavoidable.
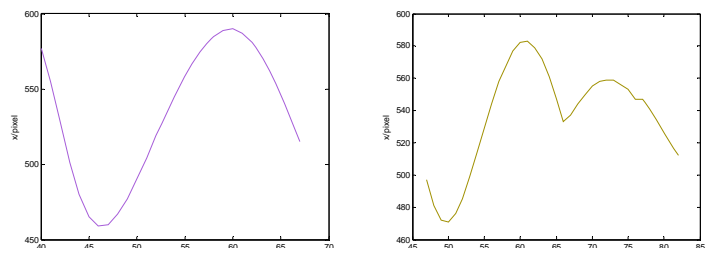

Figure 5. Typical associations: (a) Correct association. (b) False association. 
We use constrains of acceleration to break unreasonable tracks. A track is broken at a time instance if the acceleration on $\mathrm{x}$ axis is beyond five times of the average acceleration of that instance. As shown in figure 6, the trajectories are small, broken segments afterwards.

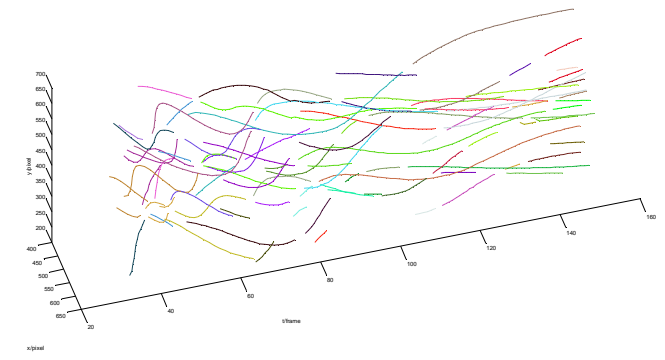

Figure 6. Break unreasonable associations.

\section{B. Trajectory Modelling}

Two non-overlapping tracks of which the gap region's changing rate of $\mathrm{y}$ axis is less than 6 pixel/frame are selected as linking candidates.
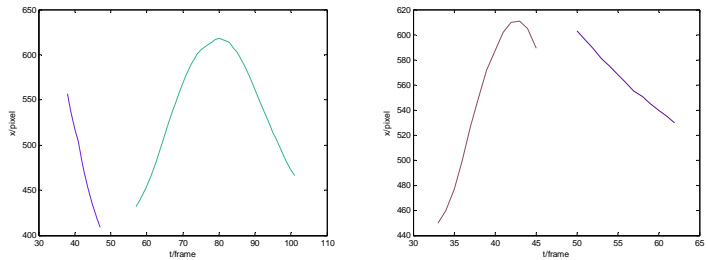

Figure 7. Linking candidates: (a) Positive linking candidates. (b) Negative linking candidates.

Linking candidates in figure 7(a) belong to the same moving particle. We can find that the linking of the two tracks is smooth which is in accordance with human intuition. Linking candidates in figure 7(b) belong to two different particles and the linking is unsmooth.

A damped vibration signal is used to describe the particle movement on $\mathrm{x}$ axis:

$$
f_{x}(t)=A e^{-a t} \sin \left(\omega e^{-b t} t+\varphi\right)+c .
$$

The amplitude is gradually changing and the angular velocity is decreasing. The changing of amplitude and angular velocity is described by exponential function.

Use equation (1) to fit the gap region of two candidate tracks. Assume there are $L$ trajectories: $\left\{T_{l}\right\}, 1 \leq l \leq L$. Any two of the tracks $T_{p}=\left\langle\mathbf{p}_{j_{a}}^{a}, \cdots, \mathbf{p}_{j_{a+L_{a}-1}}^{a+L_{a}-1}\right\rangle$ and $T_{q}=$ $\left\langle\mathbf{p}_{j_{b}}^{b}, \cdots, \mathbf{p}_{j_{b+L_{b}-1}}^{b+L_{b}-1}\right\rangle$ are selected. If $\left(a+L_{a}-1\right)<b$ and

$\frac{\left|y_{j_{b}}^{b}-\mathbf{y}_{j_{a+L_{a}-1}}^{a+L_{a}-1}\right|}{\left|b-\left(a+L_{a}-1\right)\right|} \leq 6$, then the two tracks are selected as a pair of linking candidates. Choose the second half of $T_{p}$ and the first half of $T_{q}$ to construct a new point set for fitting: $P_{p, q}=\left\{\mathbf{p}_{j_{c}}^{c}, \cdots, \mathbf{p}_{j_{a+L_{a}-1}}^{a+L_{a}-1}, \mathbf{p}_{j_{b}}^{b}, \cdots, \mathbf{p}_{j_{b+L_{b}-1}}^{b+L_{b}-1}\right\}$. The number $c$ should be chosen so that $c$ is the biggest number satisfies the condition that particle velocity at $c$ and $a+L_{a}-1$ are contrary in their signs. Choose $d$ in a correspondence way. In the fitting process, we only consider positions on $\mathrm{x}$ axis, so we fit $X_{p, q}=\left\{x_{j_{c}}^{c}, \cdots, x_{j_{a+L_{a}-1}^{a+L_{a}-1}}^{a} x_{j_{b}}^{b}, \cdots, x_{j_{b+L_{b}-1}}^{b+L_{b}-1}\right\}$ to the proposed model.

The fitting equation contains six parameters: $\theta=$ [A $a \omega b \varphi c]$. Least square fitting is applied. This problem has quite a few local minimums, so appropriate initial values are requested. The six parameters have their corresponding physical meanings and we can use $X_{p, q}$ to estimate the initial values.

$A$ is amplitude and the corresponding initial value is:

$$
A_{0}=\frac{\max \left(X_{p, q}\right)-\min \left(X_{p, q}\right)}{2}
$$

$\omega$ is angular speed. Define the maximum value of $X_{p, q}$ occurred at $t_{\max }$ and the minimum value of $X_{p, q}$ occurred at $t_{\text {min }}$. The initial value of $\omega$ is:

$$
\omega_{0}=\frac{\pi}{\left|t_{\max }-t_{\min }\right|}
$$

$\varphi$ is phase and the initial value is set to:

$$
\varphi_{0}=\frac{\pi}{2}-\omega_{0} t_{\max }
$$

$C$ is dc component and the initial value is:

$$
c_{0}=\frac{\max \left(X_{p, q}\right)+\min \left(X_{p, q}\right)}{2}
$$

$a$ describes the changing of amplitude and we set $a_{0}=$ 0 . $b$ describe the changing of angular speed and we set $b_{0}=0$.
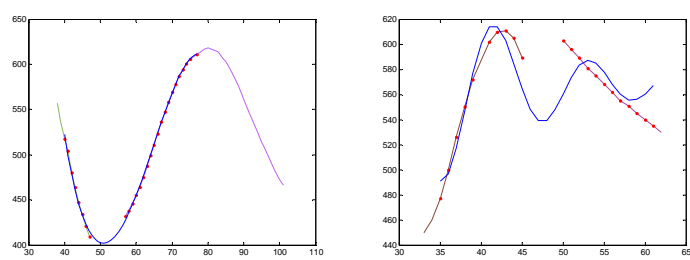

Figure 8. Gap region fitting of linking candidates.

We can conclude from figure 8 that equation (1) fits linking candidates belonging to the same particle well and fits linking candidates belonging to different particles badly. Fitting residuals can be used as features of the SVM.

\section{SVM for linking track segments}

We use SVM [6] to determine whether two linking candidate tracks should be linked. This step is called reassociation. Three values obtained from gap region fitting are used as features of the SVM. The three values are mean square residual of fitting $\bar{r}$, absolute value of fitting error on the two discontinuous points $r_{1}$ and $r_{2}$. Time length of the gap $\Delta t$ is also a feature. $\bar{r}, r_{1}, r_{2}$ and $\Delta t$ are the four features of the SVM classifier. 
179 linking candidates from 10 samples which are calibrated in manual formed the training set. SVM with RBF kernel is used as the classifier. We use LIBSVM [7] to train the classifier. Grid searching method is used to choose parameters through 5-fold cross validation. We choose penalty factor $C=8$ and kernel function parameter $\gamma=0.015625$. Thus we have a SVM to determine whether a pair of linking candidates belonging to the same trajectory.

If the output of the SVM is positive, we link the two candidate tracks to one trajectory. The linking process can be described as follows:

After the first step of nearest neighbor association and breaking unreasonable tracks, we have a set of tracks represented by $S_{T}=\left\{T_{1}, T_{2}, \cdots, T_{l}\right\}$. The number of tracks is denoted by $L$. We maintain a boolean vector $V$ with a length of $L$ to keep track of whether the corresponding track is still valid. Initialize every element of the vector to be true.

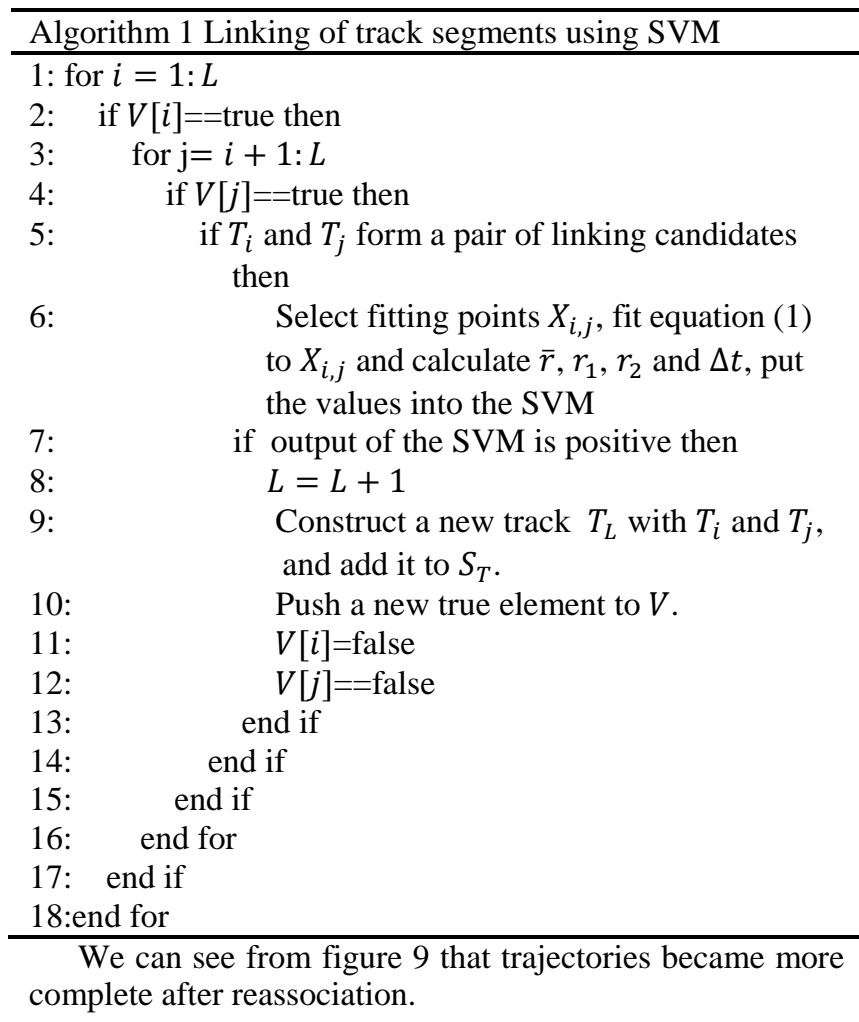

\section{COUNTING}

Our ultimate goal is counting the accurate number of visible foreign particles in liquid injection. By tracking moving particles we obtained a set of trajectories $\left\{T_{l}\right\}$. We discard trajectories whose length is less than half of the frame number $n$. The number of moving particles in the sample is the number of trajectories at last.

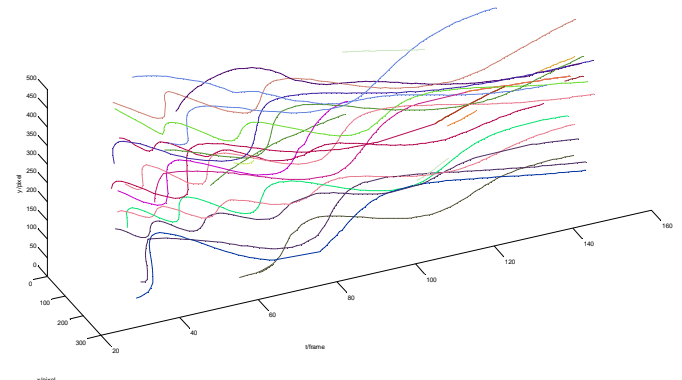

Figure 9. Trajectories after reassociation.

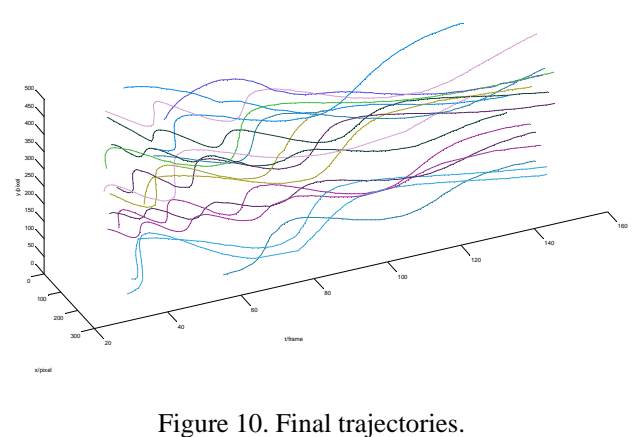

V. EXPERIMENTAL RESULTS

\section{A. Reassociation accuracy}

The training set includes 179 pair of linking candidates from 10 samples of which 89 are positive and 90 are negative. Set $C=8$ and $\gamma=0.015625$, and use 5-fold cross validation. The accuracy of classification is $96.4 \%$ and we can conclude that the classifier is effective to linking track segments.

\section{B. Counting accuracy}

Use this approach to count the numbers of 47 samples which are calibrated in manual and contain 0-20 moving particles. Samples which are count completely correct account for $78.05 \%$. All samples that are not correctly count are with deviation of 1 . We can find that the counting accuracy of moving particles is high.

\section{CONCLUSION}

Our approach of counting visible foreign particles uses fitting residuals as features for the SVM of linking tracks. The damped vibration fitting function is summarized from the moving pattern of the moving particle. We applied nearest neighbor method to do preliminary tracking first and break the obtained tracks at the instance of which the acceleration is out of a certain limit. After the first step, SVM with fitting residuals as features is used to link candidate linking tracks. Our approach utilizes the law of particle movement in this specific application and the adopted machine learning method can learn from real data. Cost functions designed by human and tedious calculation are avoided. Our approach realizes accurate counting of 
visible foreign particles in liquid injection and at the same time can assure efficiency.

\section{REFERENCES}

[1] Veenman, Cor J., Marcel JT Reinders, and Eric Backer. "Resolving motion correspondence for densely moving points." Pattern Analysis and Machine Intelligence, IEEE Transactions on 23.1 (2001): 54-72.

[2] Fortmann, Thomas E., Yaakov Bar-Shalom, and Molly Scheffe. "Sonar tracking of multiple targets using joint probabilistic data association." Oceanic Engineering, IEEE Journal of 8.3 (1983): 173184.

[3] Reid, Donald B. "An algorithm for tracking multiple targets." Automatic Control, IEEE Transactions on 24.6 (1979): 843-854.
[4] Zivkovic, Zoran. "Improved adaptive Gaussian mixture model for background subtraction." Pattern Recognition, 2004. ICPR 2004. Proceedings of the 17th International Conference on. Vol. 2. IEEE, 2004.

[5] Zivkovic, Zoran, and Ferdinand van der Heijden. "Efficient adaptive density estimation per image pixel for the task of background subtraction." Pattern recognition letters 27.7 (2006): 773-780.

[6] Cortes, Corinna, and Vladimir Vapnik. "Support-vector networks." Machine learning 20.3 (1995): 273-297.

[7] Chang, Chih-Chung, and Chih-Jen Lin. "LIBSVM: a library for support vector machines." ACM Transactions on Intelligent Systems $\begin{array}{lllll}\text { and } & \text { Technology } & \text { (TIST) } & 2.3 & \text { (2011): }\end{array}$ 